\title{
Integrating Environmental Engineering and Sustainability into Engineering Science and General Engineering Programs
}

\section{Dr. Brenda Read-Daily, Elizabethtown College}

Brenda Read-Daily received a B.S. in Civil Engineering at Bradley University, a M.S. in Environmental Engineering at the University of Notre Dame, and a Ph.D. in Environmental Engineering also at the University of Notre Dame. During her graduate studies, she conducted multidisciplinary research combining microbiology with engineering design. Her research interests include optimizing biological nutrient removal processes in agricultural drainage and wastewater treatment processes in order to mitigate pollution. She is currently developing undergraduate research opportunities examining nitrogen removal in tile drainage systems. 
Integrating Environmental Engineering and Sustainability into Engineering Science and General Engineering Programs 


\section{Introduction}

Environmental engineering has undergone major changes in the past two decades. Of the fourteen grand challenges listed by the National Academy of Engineers, three are related to environmental engineering and sustainability: providing clean water, managing the nitrogen cycle, and improving urban infrastructure. However, despite the obvious importance and recent growth of environmental engineering, it has had a bit of an identity crises over the years ${ }^{1,2}$. Environmental engineering started out as an offshoot of civil engineering with the label of sanitary engineering but overtime evolved to be its own distinct field ${ }^{1}$. It has also become a highly multidisciplinary field where many different areas of engineering and science combine ${ }^{3,4}$. Interestingly, environmental engineering is increasingly being integrated into other engineering disciplines such as mechanical and chemical where there is a heavier emphasis on pollution prevention and resource reduction ${ }^{1}$. Given the current trends, environmental engineering is an integral part of sustainability education. At its core environmental engineering is an important part of sustainability. Within the field itself, environmental engineering has further embraced the sustainability by incorporating the triple bottom line (environment, society, and economics) ${ }^{3}$.

A search of ABET-accredited programs reveals that there are currently sixty-four environmental engineering programs. In addition to these programs, there are many additional civil engineering curriculums where environmental engineering is offered as a concentration. Despite these numbers, environmental and civil engineering programs offering environmental engineering concentrations pale in comparison to those for other engineering disciplines such a civil (no environmental concentration), chemical, electrical, or mechanical. While these distinctions separate one discipline from another, there is a growing trend for more broad-based and multidisciplinary engineering.

In particular, general engineering and engineering science programs offer broad-based engineering programs. These programs are largely found at small private liberal arts institutions that do not have the numbers to support the numerous engineering disciplines ${ }^{5}$. They provide students with an ABET-accredited engineering degree rooted in fundamental engineering courses, but also allow students immerse themselves in the humanities. The graduates from these types of programs are a small, but growing portion of the overall pool of matriculating engineering students 5 . However, the number of engineering science programs has increased by $68 \%$ over the past thirteen years. Despite the general nature of engineering science, many of these programs offer concentrations in specific disciplines so students receive sufficient specialization to find jobs in the desired field ${ }^{5}$.

This research focuses on the growing number of general engineering and engineering science programs offering environmental engineering concentrations with some integrating sustainability into their curriculums. This paper provides an overview of what classes existing general engineering programs offer within their environmental engineering concentrations. As the field of engineering shift towards placing a heavier emphasis on sustainability, it is important to start a broader conversation about how general engineering and engineering science programs can integrate environmental engineering to equip future engineers. 


\section{Methodology}

Using the Accredited Program Search tool at ABET's website, a search was conducted for all the programs offering engineering science or general engineering programs with Bachelor's degrees in the United States. Sixty-four schools had engineering science degrees and two had engineering degrees. Of the schools offering engineering science degrees, any schools with separate departments dedicated to different branches of engineering were eliminated. For instance the University of Illinois at Urbana Champaign has an engineering science degree option, but it also has separate departments for Civil and Environmental Engineering, Mechanical Engineering, Electrical Engineering etc. The schools that were selected for this paper were ones that housed their engineering science or general engineering degrees within one engineering department. While Taylor University and Tarleton State University offer a separate bachelor of science in environmental engineering, they were included in the analysis because the remainder of the program is a general engineering program where students receive a Bachelor of Science or Bachelor of Engineering, and environmental engineering is the only separate bachelor's degree.

After determining the relevant general or engineering science programs, department websites and college course catalogs were checked to determine which concentrations were offered within each program. These results were compiled in a spreadsheet, and the programs offering environmental, civil, or other related type of engineering were further evaluated by examining course plans. The course plans were checked for what specific environmental engineering and sustainability-related curriculum was being offered as a part of the engineering curriculum. Specifically, course titles and course descriptions were searched for the key word "sustainability" or sustainability themes.

\section{Results and Discussion}

In the United States there are sixty-four institutions offering ABET-accredited engineering or engineering science degrees with forty-two housing engineering science programs within a single engineering department (Tables 1-3). Engineering science or general engineering programs tend to be found at smaller institutions. Privately funded institutions made up a majority (69\%) of these schools (Tables 1 and 2 ). Of the engineering departments offering engineering science degrees, only four $(9.5 \%)$ offer environmental engineering concentrations: Dartmouth College, Geneva College, Mercer University, and Messiah College (Tables 1 and 2). Notably, all of these schools are private institutions. In addition to these, Calvin College and Swarthmore College provide civil/environmental engineering concentrations. Another ten $(23.8 \%)$ schools offer concentrations in civil engineering. These findings are contrasted to the number of schools offering computer, electrical, or mechanical engineering concentrations: sixteen $(38.1 \%)$, twenty-eight $(66.7 \%)$, thirty (71.4\%), respectively (Tables $1-3)$. This result is not surprising given that environmental engineering programs are outnumbered by mechanical, electrical, and computer engineering programs nationally.

Looking more specifically at curriculums offered at these schools, they all shared common engineering cores of courses in physics, calculus, chemistry, and mechanics. Of the sixteen schools offering civil, civil/environmental, or environmental engineering concentrations, thirteen 
offered an environmental engineering course that provided either an introduction or a survey of the field (Table 4).

Table 1. Concentrations offered at Privately Funded Universities with ABET-Accredited Engineering Science Programs

\begin{tabular}{|c|c|c|c|c|c|c|c|c|c|c|c|c|c|c|c|c|}
\hline & 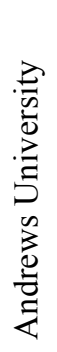 & 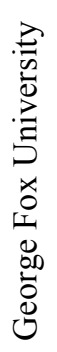 & 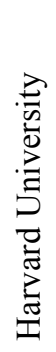 & 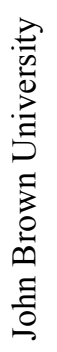 & 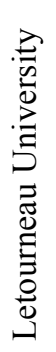 & 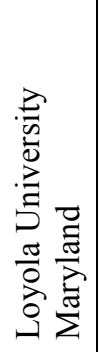 & 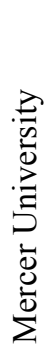 & 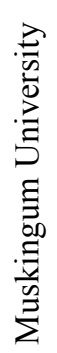 & 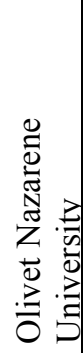 & 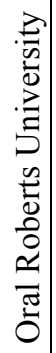 & 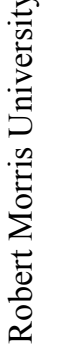 & 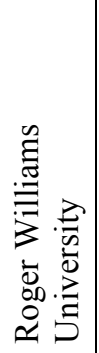 & 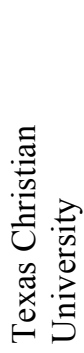 & 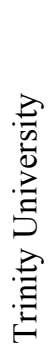 & 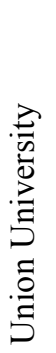 & 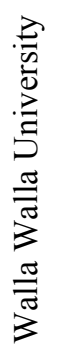 \\
\hline Biochemical & & & & & & & & & & & & & & & & \\
\hline Bioengineering & & & & & & & & & & & & & & & & \\
\hline Biomedical & & & $\mathrm{X}$ & & $\mathrm{X}$ & & X & & & $\mathrm{X}$ & $\mathrm{X}$ & & & & & \\
\hline Bioprocess & & & & & & & & & & & & & & & & \\
\hline Biotechnology & & & & & & & & & & & & & & & & \\
\hline Chemical & & & & & & & & & & & & & & $\mathrm{X}$ & & \\
\hline Civil & & $\mathrm{X}$ & & & $\mathrm{X}$ & & & & & & & & & & & $\mathrm{X}$ \\
\hline Computer & * & $\mathrm{X}$ & & & & $\mathrm{X}$ & $\mathrm{X}$ & & $\mathrm{X}$ & $\mathrm{X}$ & & & & & & $\bar{X}$ \\
\hline Electrical & * & $\mathrm{X}$ & $\mathrm{X}$ & & & $\mathrm{X}$ & $\mathrm{X}$ & & $\mathrm{X}$ & $\mathrm{X}$ & & & $\mathrm{X}$ & $\mathrm{X}$ & $\mathrm{X}$ & $\mathrm{X}$ \\
\hline $\begin{array}{l}\text { Engineering } \\
\text { Management }\end{array}$ & & & & & & & & & & & & & & & & \\
\hline Environmental & & & $\mathrm{X}$ & & & & $\mathrm{X}$ & & & & & & & & & \\
\hline Geological & & & & & & & & & $\mathrm{X}$ & & & & & & & \\
\hline Industrial & & & & & & & $\mathrm{X}$ & & & & $\mathrm{X}$ & & & & & \\
\hline Manufacturing & & & & & & & & & & & & & & & & \\
\hline Materials & & & & & & $\mathrm{X}$ & & & & & & & & & & \\
\hline Mechanical & $\mathrm{X}$ & $\mathrm{X}$ & $\mathrm{X}$ & & & $\mathrm{X}$ & $\mathrm{X}$ & & $\mathrm{X}$ & $\mathrm{X}$ & $\mathrm{X}$ & & $\mathrm{X}$ & $\mathrm{X}$ & $\mathrm{X}$ & $\mathrm{X}$ \\
\hline $\begin{array}{l}\text { Renewable } \\
\text { Energy }\end{array}$ & & & & $\mathrm{X}$ & & & & & & & & & & & & \\
\hline Robotics & & & & & & & & & & & & & & & & \\
\hline Software & & & & & & & & $\mathrm{X}$ & & & $\mathrm{X}$ & & & & & \\
\hline $\begin{array}{l}\text { Sustainable } \\
\text { Design }\end{array}$ & & & & & & & & & & & & & & & & \\
\hline None & & & & & & & & & & & & $\mathrm{X}$ & & & & \\
\hline
\end{tabular}

*Concentration offered in conjunction with another discipline for example, civil and environmental engineering concentration. 
Table 2. Concentrations offered at Privately Funded Colleges with ABET-Accredited Engineering Science Programs

\begin{tabular}{|c|c|c|c|c|c|c|c|c|c|c|c|c|c|}
\hline & 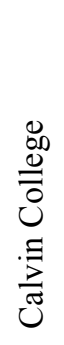 & 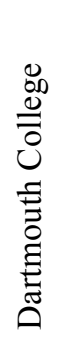 & 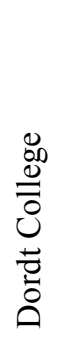 & 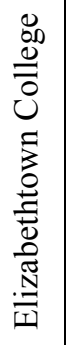 & 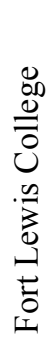 & 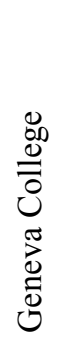 & 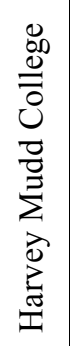 & $\begin{array}{l}0 \\
0 \\
\stackrel{0}{0} \\
0 \\
0 \\
0 \\
0 \\
0 \\
0\end{array}$ & 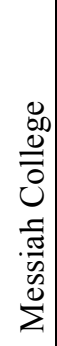 & 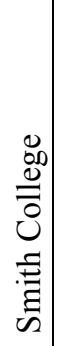 & 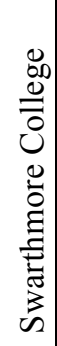 & 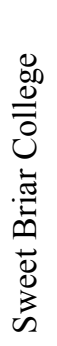 & 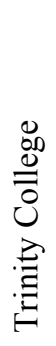 \\
\hline Biochemical & & & & & & & & $X$ & & & & & \\
\hline \multicolumn{14}{|l|}{ Bioengineering } \\
\hline Biomedical & & $\mathrm{X}$ & $\mathrm{X}$ & & & & & & $\mathrm{X}$ & & & & $X$ \\
\hline Bioprocess & & $\mathrm{X}$ & & & & & & & & & & & \\
\hline Biotechnology & & $\mathrm{X}$ & & & & & & & & & & & \\
\hline Chemical & $\mathrm{X}$ & $\mathrm{X}$ & $\mathrm{X}$ & & & $\mathrm{X}$ & & $\mathrm{X}$ & & & & & \\
\hline Civil & $*$ & & $\mathrm{X}$ & & & $\mathrm{X}$ & & & $\mathrm{X}$ & & $*$ & & \\
\hline Computer & $\mathrm{X}$ & $\mathrm{X}$ & & & & & & $\mathrm{X}$ & $\mathrm{X}$ & & & & $\mathrm{X}$ \\
\hline Electrical & $\mathrm{X}$ & $\mathrm{X}$ & $\mathrm{X}$ & $\mathrm{X}$ & & $\mathrm{X}$ & & $\mathrm{X}$ & $\mathrm{X}$ & & $X$ & & $\mathrm{X}$ \\
\hline $\begin{array}{l}\text { Engineering } \\
\text { Management }\end{array}$ & & $\mathrm{X}$ & & & & & & & & & & & \\
\hline Environmental & $*$ & $\mathrm{X}$ & & & & $\mathrm{X}$ & & & $\mathrm{X}$ & & $*$ & & \\
\hline \multicolumn{14}{|l|}{ Geological } \\
\hline \multicolumn{14}{|l|}{ Industrial } \\
\hline \multicolumn{14}{|l|}{ Manufacturing } \\
\hline Materials & & $\mathrm{X}$ & & & & & & & & & & & \\
\hline Mechanical & $X$ & $\mathrm{X}$ & $\mathrm{X}$ & $\mathrm{X}$ & & $\mathrm{X}$ & & $\mathrm{X}$ & $X$ & & $X$ & & $\mathrm{X}$ \\
\hline \multicolumn{14}{|l|}{$\begin{array}{l}\text { Renewable } \\
\text { Energy }\end{array}$} \\
\hline \multicolumn{14}{|l|}{ Robotics } \\
\hline Software & & & & & & & & & & & & $\mathrm{X}$ & \\
\hline $\begin{array}{l}\text { Sustainable } \\
\text { Design }\end{array}$ & & & & $\mathrm{X}$ & & & & & & & & & \\
\hline None & & & $\mathrm{X}$ & & $\mathrm{X}$ & & $\mathrm{X}$ & & & $X$ & & & \\
\hline
\end{tabular}

*Concentration offered in conjunction with another discipline for example, civil and environmental engineering concentration. 
Table 3. Concentrations offered at Publically Funded Universities with ABET-Accredited Engineering Science Programs

\begin{tabular}{|c|c|c|c|c|c|c|c|c|c|c|c|c|c|}
\hline & 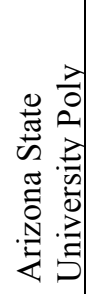 & 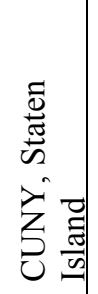 & 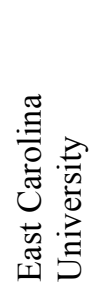 & 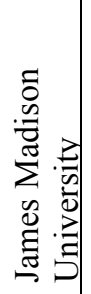 & 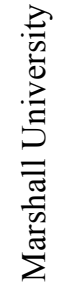 & 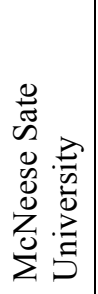 & 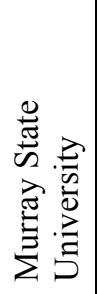 & 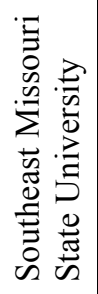 & 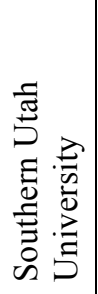 & 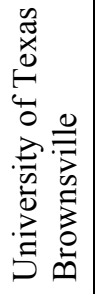 & 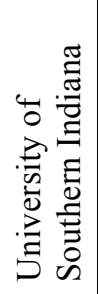 & 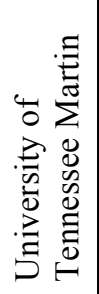 & 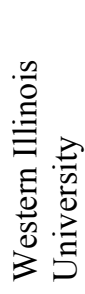 \\
\hline \multicolumn{14}{|l|}{ Biochemical } \\
\hline Bioengineering & & & & & & & & & & $\mathrm{X}$ & & & \\
\hline Biomedical & & & $\mathrm{X}$ & & & & $\mathrm{X}$ & & & & & & \\
\hline \multicolumn{14}{|l|}{ Bioprocess } \\
\hline \multicolumn{14}{|l|}{ Biotechnology } \\
\hline Chemical & & & & & & $\mathrm{X}$ & & & & & & & \\
\hline Civil & $\mathrm{X}$ & & & & $\mathrm{X}$ & $\mathrm{X}$ & & & & & & $\mathrm{X}$ & $X$ \\
\hline Computer & & $\mathrm{X}$ & & & & & & $\mathrm{X}$ & & $\mathrm{X}$ & & & * \\
\hline Electrical & $\mathrm{X}$ & $\mathrm{X}$ & $\mathrm{X}$ & & & & $\mathrm{X}$ & $\mathrm{X}$ & & $\mathrm{X}$ & & $\mathrm{X}$ & $*$ \\
\hline \multicolumn{14}{|l|}{$\begin{array}{l}\text { Engineering } \\
\text { Management } \\
\end{array}$} \\
\hline \multicolumn{14}{|l|}{ Environmental } \\
\hline \multicolumn{14}{|l|}{ Geological } \\
\hline Industrial & & & $\mathrm{X}$ & & & & & & & & & $\mathrm{X}$ & $\mathrm{X}$ \\
\hline \multirow{2}{*}{\multicolumn{14}{|c|}{ Manufacturing }} \\
\hline & & & & & & & & & & & & & \\
\hline Mechanical & $\mathrm{X}$ & $\mathrm{X}$ & $\mathrm{X}$ & & & $X$ & $\mathrm{X}$ & $\mathrm{X}$ & & $\mathrm{X}$ & & $X$ & $\mathrm{X}$ \\
\hline \multicolumn{14}{|l|}{$\begin{array}{l}\text { Renewable } \\
\text { Energy }\end{array}$} \\
\hline Robotics & $\mathrm{X}$ & & & & & & & & & & & & \\
\hline \multicolumn{14}{|l|}{ Software } \\
\hline \multicolumn{14}{|l|}{$\begin{array}{l}\text { Sustainable } \\
\text { Design }\end{array}$} \\
\hline None & & & & $\mathrm{X}$ & & & & & $\mathrm{X}$ & & $\mathrm{X}$ & & $\mathrm{X}$ \\
\hline
\end{tabular}

*Concentration offered in conjunction with another discipline for example, civil and environmental engineering concentration. 
Table 4. Environmental Engineering Concentration Electives Offered at ABET-accredited Engineering Science and General Engineering Programs

\begin{tabular}{|c|c|c|c|c|c|c|c|c|}
\hline Institution & 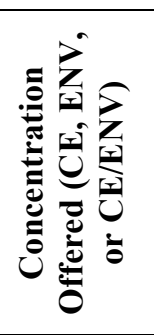 & 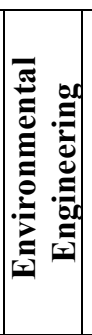 & 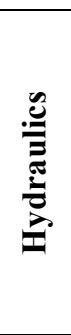 & 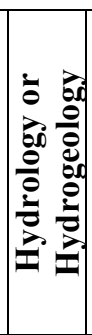 & 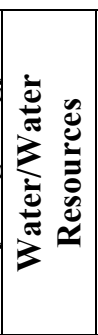 & 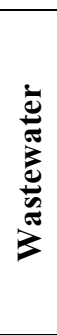 & 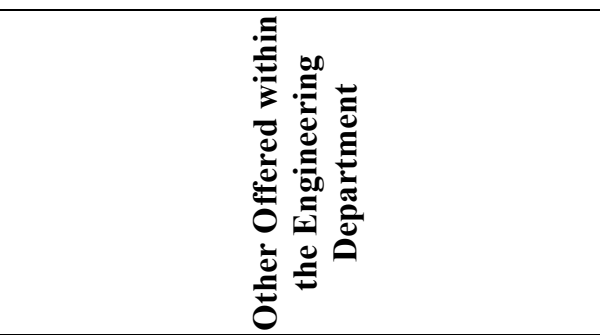 & 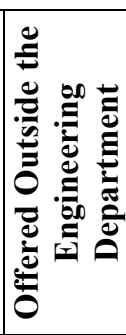 \\
\hline $\begin{array}{l}\text { Arizona State } \\
\text { University } \\
\text { Polytechnic } \\
\end{array}$ & $\mathrm{CE}$ & & & & $\mathrm{X}$ & $\mathrm{X}$ & $\begin{array}{l}\text { Solid Waste, Environmental Systems } \\
\text { Project }\end{array}$ & \\
\hline Calvin College & $\mathrm{CE} / \mathrm{ENV}$ & $\mathrm{X}$ & $\mathrm{X}$ & & & & Environmental Engineering Design & \\
\hline $\begin{array}{l}\text { Dartmouth } \\
\text { College }\end{array}$ & ENV & $\mathrm{X}$ & & & & & $\begin{array}{l}\text { Sustainable Design, Sustainable } \\
\text { Resource Engineering }\end{array}$ & \\
\hline Dordt College & $\mathrm{CE}$ & $\mathrm{X}$ & & & & & & \\
\hline Geneva College & ENV & $\mathrm{X}$ & $\mathrm{X}$ & $\mathrm{X}$ & $*$ & $*$ & $\begin{array}{l}\text { Environmental Monitoring, Transport } \\
\text { Processes, Air Pollution Control, Solid } \\
\text { and Hazardous Waste }\end{array}$ & $\mathrm{X}$ \\
\hline $\begin{array}{l}\text { George Fox } \\
\text { University }\end{array}$ & $\mathrm{CE}$ & $\mathrm{X}$ & & & $\mathrm{X}$ & & & \\
\hline $\begin{array}{l}\text { LeTourneau } \\
\text { University }\end{array}$ & $\mathrm{CE}$ & $\mathrm{X}$ & $\mathrm{X}$ & $\mathrm{X}$ & $*$ & $*$ & & \\
\hline $\begin{array}{l}\text { McNeese State } \\
\text { University }\end{array}$ & $\mathrm{CE}$ & & $\mathrm{X}$ & $\mathrm{X}$ & & $\mathrm{X}$ & Pollution Control & \\
\hline Mercer University & $\mathrm{CE}$ & $\mathrm{X}$ & $\mathrm{X}$ & $\mathrm{X}$ & & $\mathrm{X}$ & Public Health & \\
\hline Messiah College & ENV & $\mathrm{X}$ & & $\mathrm{X}$ & & & & $\mathrm{X}$ \\
\hline $\begin{array}{l}\text { Harvey Mudd } \\
\text { College }\end{array}$ & None & $\mathrm{X}$ & & & & & $\begin{array}{l}\text { Mass Transfer and Separation Processes, } \\
\text { Transport Phenomena }\end{array}$ & \\
\hline $\begin{array}{l}\text { Harvard } \\
\text { University }\end{array}$ & ENV & $\mathrm{X}$ & & $X$ & $\mathrm{X}$ & & & \\
\hline $\begin{array}{l}\text { Marshall } \\
\text { University }\end{array}$ & $\mathrm{CE}$ & & & & $\mathrm{X}$ & $\mathrm{X}$ & & \\
\hline $\begin{array}{l}\text { Swarthmore } \\
\text { College }\end{array}$ & $\mathrm{CE} / \mathrm{ENV}$ & $\mathrm{X}$ & & & $\mathrm{X}$ & & & \\
\hline $\begin{array}{l}\text { Tarleton State } \\
\text { University* }\end{array}$ & ENV & $X$ & & $X$ & $X$ & $X$ & $\begin{array}{l}\text { Environmental Systems Modeling, Texas } \\
\text { Water Management }\end{array}$ & \\
\hline $\begin{array}{l}\text { Taylor } \\
\text { University* }\end{array}$ & ENV & & & & & & & $X$ \\
\hline $\begin{array}{l}\text { University of } \\
\text { Tennessee at } \\
\text { Martin }\end{array}$ & $\mathrm{CE}$ & & $*$ & $*$ & & & & \\
\hline $\begin{array}{l}\text { Walla Walla } \\
\text { University }\end{array}$ & $\mathrm{CE}$ & $X$ & & $X$ & & $X$ & & \\
\hline $\begin{array}{l}\text { Western IL } \\
\text { University }\end{array}$ & $\mathrm{CE}$ & & & & & & & \\
\hline
\end{tabular}

*Topics in both subjects were covered in one course. 
Courses in hydrology, hydraulics, water resources management, or wastewater were also common to the civil or environmental engineering concentrations as is the case in traditional environmental engineering programs. Interestingly, several of the schools with civil engineering concentrations offered more environmental engineering courses than schools with environmental engineering concentrations. LeTourneau University, for example, offers a concentration in civil engineering, but within that concentration, students can further specialize in water resources. This is evidenced by its selection of courses including an introductory environmental engineering course, hydraulics, hydrology, and water resources, and wastewater. Other schools rely more heavily upon curriculum offered outside of their engineering department as environmental engineering electives such as: organic chemistry, biochemistry, environmental science, geology, and ecology. Taylor University and Messiah College, for example, were schools that satisfied most of their requirements for the environmental engineering concentration or major outside of their departments. Harvey Mudd College does not specifically offer an environmental engineering concentration, but students are able to take environmental engineering courses as electives.

While it is apparent that a variety of environmental engineering courses are offered within environmental engineering concentrations, few schools had classes that had the word "sustainability" in the course title. Dartmouth College offers courses in Sustainable Design and Sustainable Resource Management (Table 1). Even upon closer examination at course descriptions, few provided specific topics of sustainability or mentioned typical sustainability themes going beyond environmental protection. This result is similar to what Woolschlager (2013) found when he examined sixty ABET-accredited environmental engineering programs on their level of sustainability integration based on metrics of mentions of sustainability or sustainability concepts in program education objectives, student outcomes, courses, and department websites. Only 7\% had a strong integration of sustainability. Upon looking closer, at all programs some merely paid lip service to sustainability while others such as Columbia University, Cornell University, Northwestern University, and South Dakota School of Mines made transformational changes to curriculum in order emphasize sustainability ${ }^{6}$.

For those programs that are already offering environmental engineering as a concentration, further work can be done to enhance the connections to other disciplines using sustainability. Modification or adaptation of traditional courses in environmental engineering may be an easy way to bridge these gaps. Most programs offer some type of introduction or survey of environmental engineering, and such a course may provide an ideal spot to include the themes of social and economic impacts of engineering solutions. Additionally, more specialized courses in topics such as water and wastewater treatment can emphasize resource choices and designs in light of societal and economic impact. Increased focus on the triple bottom line as opposed to the environmental outcomes only is necessary in the rapidly changing field. In particular, general engineering and engineering science programs can capitalize on this growing trend by carefully weaving environmental engineering coursework with a particular emphasis on sustainability into their curriculums.

\section{Conclusions}


Environmental engineering has undergone major changes in the past few decades, arguably with the most important being the integration sustainability and heavier emphasis on multidisciplinary solutions. Given these trends, general engineering and engineering science programs have a unique opportunity to capitalize on this trend, particularly those schools offering environmental engineering concentrations. Based on this study, there is room for more general engineering and engineering science programs to provide environmental engineering concentrations as only $9.5 \%$ currently do so. This $9.5 \%$ comes exclusively from privately-funded colleges and universities. A closer look at the programs offering environmental engineering curriculums showed that few specifically focus on sustainability in their courses based on course descriptions and titles. Like those programs with majors in environmental engineering, general or engineering science programs can improve their current curriculums by incorporating themes of sustainability within traditional courses and thus establish more links to other disciplines. Given the strong interest in the environment and sustainability by many students, engineering science and general engineering programs may find adding an environmental engineering concentration an attractive option. Current programs offering environmental engineering concentrations should consider ways to increase the coverage of sustainability themes and topics within their traditional courses.

\section{Bibliography}

1. Lynch, J. 2006. Recruiting for the Environmental Engineering Profession: Improving the Image of the Discipline. Proceedings of the 2006 American Society for Engineering Education Annual Conference \& Exhibition, Chicago IL, June 18-21.

2. Workshop on the Evolution of Environmental Engineering as a Professional Discipline. Toronto, Ontario, Canada, August 9-10, 2002.

3. Mihelcic, J.R., Crittenden, J.C., Small, D.R., Hokanson, D.R., Zhang, Q., Chen, H., Sortby, S.A., James, V.U., Sutherland, J.W., and Schnoor, J.L. 2003. Sustainability Science and Engineering: The Emergence of a New Metadiscipline, Environmental Science and Technology, 37, 5314-5324.

4. Young, T., Powers, S., Collins, A., and Ackerman, N. 1996. A Unified Elective Concentration in Environmental Engineering. Proceedings of the 1996 American Society for Engineering Education Annual Conference \& Exhibition, Washington, D.C, June 23-26.

5. Newberry, B. and Farison, J. 2003. A Look at the Past and Present of General Engineering and Engineering Science Programs. Journal of Engineering Education, 92(3), 217-224.

6. Woolschlager, J. 2013. Implementation of Sustainability Concepts in Environmental Engineering Curriculums, Proceedings of the 2013 American Society for Engineering Education Annual Conference \& Exhibition, Atlanta, GA, June 23-26. 